Published in final edited form as:

Mol Psychiatry. 2020 September ; 25(9): 2119-2129. doi:10.1038/s41380-018-0276-1.

\title{
Individual-specific functional connectivity markers track dimensional and categorical features of psychotic illness
}

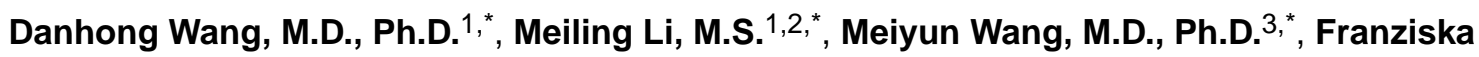 \\ Schoeppe, M.D. ${ }^{1}$, Jianxun Ren, B.S. ${ }^{1}$, Huafu Chen, Ph.D. ${ }^{2}$, Dost Öngür, M.D., Ph.D. ${ }^{4}$, Justin \\ T. Baker, M.D., Ph.D.\#4,\#, Hesheng Liu, Ph.D. \#1,3,5,6,\# \\ ${ }^{1}$ Athinoula A. Martinos Center for Biomedical Imaging, Department of Radiology, Massachusetts \\ General Hospital, Harvard Medical School, Charlestown, MA, USA \\ ${ }^{2}$ Key Laboratory for Neurolnformation of Ministry of Education, School of Life Science and \\ Technology, University of Electronic Science and Technology of China, Chengdu, China \\ ${ }^{3}$ Department of Radiology, Henan Provincial People's Hospital, Zhengzhou, China \\ ${ }^{4}$ Psychotic Disorders Division, McLean Hospital, Harvard Medical School, Belmont, MA, USA \\ Institute for Research and Medical Consultations, Imam Abdulahman Bin Faisal University, \\ Dammam, Saudi Arabia \\ ${ }^{6}$ Beijing Institute for Brain Disorders, Capital Medical University, Beijing, China \\ \# These authors contributed equally to this work.
}

\begin{abstract}
Neuroimaging studies of psychotic disorders have demonstrated abnormalities in structural and functional connectivity involving widespread brain networks. However, these group-level observations have failed to yield any biomarkers that can provide confirmatory evidence of a patient's current symptoms, predict future symptoms, or predict a treatment response. Lack of precision in both neuroanatomical and clinical boundaries have likely contributed to the inability of even well-powered studies to resolve these key relationships. Here, we employed a novel approach to defining individual-specific functional connectivity in 158 patients diagnosed with schizophrenia $(n=49)$, schizoaffective disorder $(n=37)$ or bipolar disorder with psychosis $(n=$ 72 ), and identified neuroimaging features that track psychotic symptoms in a dimension- or disorder-specific fashion. Using individually-specified functional connectivity, we were able to estimate positive, negative, and manic symptoms that showed correlations ranging from $r=0.35$ to $r=0.51$ with the observed symptom scores. Comparing optimized estimation models among
\end{abstract}

Users may view, print, copy, and download text and data-mine the content in such documents, for the purposes of academic research, subject always to the full Conditions of use:http://www.nature.com/authors/editorial_policies/license.html\#terms

Address correspondence to: Dr. Hesheng Liu, Athinoula A. Martinos Center for Biomedical Imaging, Massachusetts General Hospital/ Harvard Medical School, 149 13th St., Suite 2301, Charlestown, MA 02129, USA; hesheng@nmr.mgh.harvard.edu, Dr. Justin Baker, McLean Hospital, 115 Mill Street, Belmont, MA 02478; jtbaker@partners.org.

*Co-first Authors

\# $_{\text {Co-senior Authors }}$

CONFLICT OF INTEREST

The authors declare no conflict of interest.

Supplementary information is available at MP's website. 
schizophrenia spectrum patients, positive and negative symptoms were associated with largely non-overlapping sets of cortical connections. Comparing between schizophrenia spectrum and bipolar disorder patients, the models for positive symptoms were largely non-overlapping between the two disorder classes. Finally, models derived using conventional region definition strategies performed at chance levels for most symptom domains. Individual-specific functional connectivity analyses revealed important new distinctions among cortical circuits responsible for the positive and negative symptoms, as well as key new information about how circuits underlying symptom expressions may vary depending on the underlying etiology and illness syndrome from which they manifest.

\section{Keywords}

psychosis; bipolar disorder; schizophrenia; functional connectivity; psychoradiology

\section{INTRODUCTION}

Brain-based biomarkers with sufficient sensitivity and specificity to track with specific symptoms at the level of individuals would have the potential to radically transform the assessment and management of patients with psychotic disorders. These patients, who are in varying states of illness, may lose the ability to portray their symptom burden, which has tremendous inter-individual heterogeneity in cross-sectional presentations and complex symptom trajectories over time within individuals. Use of brain imaging, including structural and functional MRI, has shown great promise for identifying meaningful biomarkers in mental disorders, and has increasingly identified macroscopic structures that may differentiate broad disease categories. For example, studies of psychotic disorders have found abnormalities in functional connectivity involving widespread cortical and subcortical brain networks ${ }^{1-10}$, as well as irregularities in brain structure such as ventricular enlargement and cortical thinning ${ }^{11}$, some of which may scale with the intensity of ongoing symptoms ${ }^{12-16}$. Nevertheless, these findings have yet to cohere into a set of regional interactions that reliably and reproducibly track with a patient's current symptom burden in a manner that has shown successful application in the assessment of individual patients.

The study of circuit abnormalities in psychosis may have been hampered by the lack of precision in mapping functional brain regions in individual patients. Large-scale studies of human brain organization have yielded stable parcellations of the human cerebral cortex ${ }^{17-21}$ and subcortical structures ${ }^{22-24}$, providing a new cortical taxonomy for describing regional and network-level disruptions in cortical function contingent with disease.

However, these atlases only reflect general principles of functional organization in a population, rather than the idiosyncrasies of an individual. Until now, most imaging studies have relied on applying group-level atlases when assessing individual-level functional data. While these group-level analyses have revealed important relationships between connectivity and clinical characteristics (e.g. ${ }^{25,26}$ ), subtle relations could be missed if the symptom expressions are related to functional networks that are highly variable across individuals, especially those in the association cortices ${ }^{27,28}$. Recent studies have indicated that important features of brain networks could be absent in group-based templates but are evident within 
individuals ${ }^{19,29}$. Applying the group-level atlas to individual subjects can thus dilute brainbehavior associations that are critical for understanding the specific disease processes. To address this challenge, our group and others have developed methods that account for individual heterogeneity in cortical functional anatomy as a strategy to improve the robustness and inter-individual reliability of functional connectivity information and its potential relationship with cognitive abilities in healthy individuals ${ }^{30}$. Nevertheless, it remains unclear whether connectivity analyses based on individually-specified regions will facilitate the discovery of robust illness surrogate markers for global and/or dimensionspecific symptom domains. To test this idea, here we examined functional connectivity in a large cohort of individuals expressing varying degrees of psychotic symptoms, and employed machine learning under a range of constraints to identify connections that track with the principal domains of psychotic symptoms (e.g., positive symptoms, negative symptoms, mania). Estimation models were optimized using both dimension-alone and categorical-dimensional assumptions about the underlying nature of pathophysiological disturbances in psychotic syndromes.

\section{MATERIALS AND METHODS}

\section{Participants}

A total of 158 patients with DSM-IV diagnosed schizophrenia (SZ, $n=49$ ), schizoaffective disorder (SZA, $n=37$ ) or bipolar disorder with psychosis (BP, $n=72$ ) were recruited from inpatient and outpatient treatment settings at McLean Hospital with informed consent. See Supplementary Materials for the inclusion criteria. Functional MRI data (1-2 scans, 372 seconds per scan) were collected on a single 3T Tim Trio scanner (Siemens Healthcare, Erlangen, Germany) at McLean Hospital, using the vendor-supplied 12-channel phasedarray head coil $\left(\mathrm{TR}=3000 \mathrm{~ms}, \mathrm{TE}=30 \mathrm{~ms}\right.$, flip angle $=85^{\circ}, 3 \times 3 \times 3 \mathrm{~mm}$ voxels $)$, while participants were instructed to lie still with their eyes open. An in-bore camera (ISCAN, $120 \mathrm{~Hz}$, infra-red illumination) monitored participant behavior and arousal state. After quality control of the data (using a slice-based signal-to-noise ratio ${ }^{31}>100$ ), data from 44 SZ patients, 32 SZA patients, and $55 \mathrm{BP}$ patients were retained for subsequent analysis. Among them, 72 subjects ( $20 \mathrm{SZ}, 23 \mathrm{SZA}$, and $29 \mathrm{BP}$ ) were reported in previous publications ${ }^{32}$. Participants' demographic and clinical characteristics are summarized in Supplementary Table S1.

\section{Imaging data preprocessing}

Resting-state fMRI data were processed using procedures previously described ${ }^{33,34}$. See Supplementary Materials.

\section{Identifying homologous functional regions of interest (ROIs) in individuals}

Functional ROIs were localized in each individual according to the following steps. First, eighteen cortical networks were mapped in each subject using the iterative parcellation approach recently developed and validated by our group ${ }^{35}$. The algorithm was initially guided by the group-level functional network atlas derived from 1000 healthy subjects and then iteratively refined network boundaries in the individual. The individual-level cortical networks were then segmented into discrete "patches" using a clustering algorithm 
(mri_surfcluster in FreeSurfer). Finally, discrete patches in individual subjects were matched to the 116 cortical ROIs extracted from the population-level atlas estimated in 1000 subjects. The template matching procedure was performed for each cortical network as follows: 1) If an individual-level patch overlapped with a single ROI in the atlas, then the patch was labeled as the same ROI in the atlas. 2) If an individual-level patch overlapped with multiple ROIs in the network, then the patch was split into multiple smaller patches. Specifically, vertices overlapping with the group-level ROIs were labeled according to these ROIs, forming the centers of several smaller patches. The remaining vertices in the original patch were then assigned to the nearest ROIs according to the geodesic distance on the brain surface. 3) If a patch did not overlap with any ROI in the group-level network, then the patch was assigned to its nearest ROI if the shortest distance between the patch and the ROI was within a certain threshold (we used mean distance between any two vertices in the nearest ROI); 4) otherwise the patch was labeled as "unrecognized". See Supplementary Materials for more details.

\section{Strategy for symptom estimation}

Resting-state functional connectivity among the individual-specific ROIs was computed, resulting in a large pairwise connectivity matrix for each subject (see Supplementary Fig. S1). A support vector machine for regression (SVR) model was trained to estimate each patient's symptom severity scores based on the connectivity among individualized ROIs. Importantly, connections sensitive to head motion were excluded and potential confounds including age and gender were controlled in the model. The leave-one-out cross validation (LOOCV) was employed, i.e., data from $N-1$ subjects were used to train the model and then the resulting model was applied to the data of the remaining subject to estimate the subject's symptom severity. The procedure was repeated $N$ times to estimate the symptom scores of all subjects. Correlation between the estimated and observed symptom scores was then evaluated. A nonparametric permutation test was performed to determine whether the correlation simply occurred by chance. Specifically, the observed symptom scores were randomly reshuffled among the subjects and then the symptom estimation procedures were repeated (1,000 permutations). The permutation $p$ value was estimated by calculating the percentage of permutations that yielded an estimation-observation correlation value higher than the estimation-observation correlation based on the real data. See Supplementary Materials for more details.

\section{Code availability}

Codes for cortical network parcellation, ROI extraction, and symptom score estimation can be downloaded from our website (http://nmr.mgh.harvard.edu/bid/DownLoad.html).

\section{RESULTS}

\section{Individually-specified functional connectome tracks PANSS positive symptoms}

Using a subject-specific, iterative functional network parcellation strategy ${ }^{30,34}$, we mapped 18 cortical networks for each subject and then extracted 116 discrete ROIs from these networks. These functional ROIs demonstrated substantial inter-individual variability across individuals (see Supplementary Fig. S2 for examples). Functional connectivity was 
evaluated among these ROIs for each individual for the exploration of brain-behavior relationship.

To determine whether individually-specified functional connectivity tracks with the positive symptoms of psychosis, we trained SVR models to estimate the PANSS positive scores from each of the individual participants carrying a SZ or SZA diagnosis. Importantly, connections sensitive to head motion were identified first and excluded (see Supplementary Materials for the procedure and Supplementary Fig. S3). Age and gender were controlled for as covariates. We found that PANSS positive symptom scores were robustly estimated by a set of functional connections, with a moderate but statistically significant correlation between estimated and observed PANSS positive scores among the 76 patients (Fig. 1a, $r=0.50, p=$ 0.004 , all p-values in this study were based on permutation tests). Connections that contributed most to the estimation of PANSS positive scores mainly involved the frontoparietal control network (FPN), dorsal attention network (ATN), and motorsensory regions (MOT) (Fig. 1b, see also Supplementary Fig. S8 for the connections positively and negatively correlated with the symptom scores, respectively). For comparison, the analysis was repeated using functional connectivity among the corresponding ROIs identified in Yeo's group-level functional network atlas ${ }^{34}$. Atlas-based connectivity was unable to estimate PANSS positive scores regardless of whether connections sensitive to head motion were excluded $(r=0.28, p=0.09$, Fig. 1c) or included in the model $(r=0.25, p=0.087$, Supplementary Fig. S4a).

\section{Functional connectivity among individually-specified ROls tracks PANSS negative symptoms}

To demonstrate the specificity of our approach to estimate symptom levels in multiple symptom domains, we next tested whether individually-specified connectivity could track with levels of negative symptoms in the same group of individuals with SZ or SZA. Here, we again found a moderate, statistically significant correlation between estimated and observed PANSS negative symptom scores $(r=0.35, p=0.033$, Fig. 2a). Connections that contributed most to the estimation of PANSS negative scores mainly involved the FPN and MOT, and the salience network (SAL, Fig. 2b \& Supplementary Fig. S8). In contrast, PANSS negative scores estimated by atlas-based connectivity (i.e., Yeo's group-level atlas) were uncorrelated with observed negative symptoms, when tested with either motion-related connections excluded $(r=0.03, p=0.403$, Fig. $2 \mathbf{c})$ or included $(r=0.02, p=0.460)$. Furthermore, the connections that contributed to negative symptom estimation models showed no overlap with the connections that contributed to positive symptom models (Supplementary Fig. S4), indicating that our approach yields sets of connections specific to particular symptom domains in largely non-overlapping brain systems.

\section{Between-network connectivity abnormality is a major contributor to positive and negative symptoms in patients with schizophrenia spectrum illness}

Inspecting the connections that were predictive of the PANSS positive and negative symptoms (e.g., Fig. 1b \& Fig. 2b), we found that the majority of them were connections between different functional networks rather than connections within the same network (see Supplementary Materials for the details of how between-network connectivity was 
estimated). Importantly, the absolute values of between-network connections were significantly reduced when ROIs were individually-specified, compared to atlas-defined (see Supplementary Fig. S5); nevertheless, the reduced connectivity can yield better symptom estimates, suggesting that between-network connectivity may be more accurately quantified when functional regions are localized in individuals.

Grouping the connections according to the 7 canonical functional networks ${ }^{34}$, we observed that connections contributing to the positive symptom estimation model were mostly between-network connections that involved the FPN, ATN, and MOT (Fig. 3a). In contrast, connections contributing to the negative symptom estimation model mainly involved the FPN and MOT (Fig. 3b).

\section{Functional connectivity estimates of symptom dimensions perform better within diagnostic groups}

While a large body of genetic, imaging, and neuropathology research suggest that SZ, SZA, and BP share some aspects of pathophysiology, it remains unclear whether similar connectivity abnormalities give rise to similar clinical presentations in patients within distinct diagnostic classes. To address this question, we trained SVR models to estimate PANSS positive and negative symptoms in 55 patients carrying a diagnosis of BP with psychosis.

As in the schizophrenia spectrum group, we found that functional connectivity among the individually-specified ROIs yielded estimates of PANSS positive subscale scores in the bipolar patients, with a mild-moderate, and nominally significant, correlation $(r=0.35, p=$ 0.048, Fig. 4). However, in contrast to the PANSS positive estimation model in the SZ/SZA group, PANSS positive symptom estimation in the bipolar group was driven mainly by between-network connectivity involving the visual network (VIS), DN, FPN and SAL, with no overlap between the connections contributing to positive symptom estimation in the two patient groups (Supplementary Fig. S6a). To further test whether positive symptoms were driven by any common connections in the two groups, we aggregated patients across both affective and non-affective psychosis and then trained SVR models to estimate PANSS positive scores. Despite this analysis employing the largest number of participants, individually-specified connectivity in this cross-diagnostic cohort did not yield PANSS positive estimates that correlated with observed positive symptom levels $(r=0.15, p=0.187$, Supplementary Fig. S6b).

In the case of PANSS negative symptoms in the BP patients, neither individually-specified ROIs $(r=0.11, p=0.261)$ nor atlas-based ROIs $(r=0.13, p=0.192)$ yielded robust symptom estimates. Although, as noted above, the PANSS negative scores could be estimated in the schizophrenia spectrum patients, the cross-diagnostic patient cohort that included BP patients yielded estimates that performed no better than chance $(r=0.20, p=$ 0.081, Supplementary Fig. S6c).

Taken together, these observations indicate that positive and negative symptoms may result from distinct pathophysiological mechanisms in affective and non-affective psychosis, which 
must be accounted for when employing connectivity-based measures in evaluating the two conditions.

\section{Functional connectivity among individually-specified ROls predicts mania scores in BP patients}

As functional regions identified in individuals may better capture the idiosyncrasies of subjects than atlas-based ROIs, performing connectivity analyses based on individuallyspecified ROIs may facilitate the discovery of meaningful brain-based biomarkers for a variety of symptoms and behaviors beyond the PANSS scales. As a proof of this concept, we trained SVR models to estimate the level of mania in the $55 \mathrm{BP}$ patients, using the same approach outlined above. Connections sensitive to head motion were identified first and excluded. We found that functional connectivity among the individually-specified ROIs yielded moderately strong correlations between estimated and observed Young Mania Rating Scale (YMRS) totals $(r=0.51, p=0.011)$. The optimized mania estimation model was driven mainly by between-network connectivity involving the SAL, VIS, LMB, and DN (Fig. 5). Connectivity within the FPN also contributed to the estimation. Again, functional connectivity among the ROIs defined by Yeo's group-level atlas did not yield YMRS estimates that correlated with observed mania levels $(r=0.17, p=0.217)$.

\section{Additional Analyses}

We performed a series of additional analyses to test if our results were affected by medication, the selection of group-level atlas, or the method for cross validation. We found that our symptom estimation models were not influenced by medication (Supplementary Fig. S7). We repeated the analyses using Glasser's group-level atlas, which consisted of 360 areas ${ }^{36}$, as well as 10 -fold cross validation, and found that our conclusions remained unchanged (see Supplementary Materials). Focusing on the symptom-related connections (as shown in Fig. 1, 2, 4 \& 5), we found that the same connections defined by group-level ROIs were less correlated with symptom scores (Supplementary Fig. S9), indicating that the symptom-related connections were obscured by the group-level atlas, thus impairing the prediction of symptoms. Finally, we performed analyses to test if macroscopic anatomical features are related to the symptom scores, and found that models using macroscopic anatomical features, such as sulcal depth and cortical thickness, were unable to estimate psychotic symptoms (Supplementary Table S2).

\section{DISCUSSION}

In this study, we identified connectivity among individually-specified functional regions that was able to yield moderate-to-strong estimates of symptom levels across several principal domains of psychotic disorder symptomatology. Importantly, without accounting for individual variation in cortical functional anatomy, conventional atlas-based connectivity was unable to predict these symptoms in almost every case. Moreover, the specific sets of network interactions predicting symptom burden changed substantially when taking our individual-specific approach, with between-network variance explaining dramatically more symptom-specific severity than in atlas-defined models. Our work highlights the critical importance of accounting for individual variation in cortical functional anatomy in 
psychoradiology research ${ }^{37-39}$ and taking a conservative approach to de-confound human neuroimaging data, particularly when the goal is to identify meaningful clinicalneurobiological correlations that could serve pragmatic clinical utility in diagnosis, prognosis, and treatment selection for patients with psychiatric disorders.

\section{A conservative neuroimaging approach facilitates robust biomarker discovery in schizophrenia and bipolar disorder}

Prior studies on psychosis have related a wide range of neuroimaging-derived metrics to several prominent symptom domains. For example, positive symptoms have been related to reduced cortical thickness and brain volume ${ }^{14,15,40}$, abnormal fractional anisotropy $41-44$, and altered resting-state as well as task-based functional connectivity, with some convergent evidence for abnormalities among fronto-temporal and limbic structures ${ }^{44-46}$. Likewise, several reports have documented correlations between negative symptom severity and cortical thinning and/or reduced gray matter ${ }^{47-49}$, particularly in the temporal and frontal lobes, basal ganglia, corpus callosum, thalamus, and the paralimbic system. Levels of mania have been related to the extent of prefrontal white matter abnormalities, as well as decreased connectivity and task-based fMRI responses involving the cingulum, the prefrontal cortex and failure of de-activation in the medial frontal cortex during active tasks ${ }^{50-53}$. The lack of specificity and reproducibility in the literature may be due to the considerable heterogeneity of clinical presentations, assessment strategies, and differences in data processing, together with a failure to sufficiently account for confounding sources of variance. These limitations may have reduced the effective power in even large-scale studies to detect true illness-related effects.

By taking a machine learning approach, our findings reflect unbiased, data-driven estimates of which sets of cortical connections allow for optimal estimation of a particular symptom summary score, independent of any other symptom domains tested. Although this approach underestimates the full set of interactions that carry illness-related information, it nevertheless demonstrates tremendous heuristic value as a tool for human network neuroscience, yielding simple, reliable readouts to test how changes in a particular model affect the model's ability to estimate a particular set of symptoms. For instance, our models were unable to estimate positive or negative psychotic symptom domains when data from SZ/SZA and BP patients were considered together. And yet, these same symptoms were estimated far more reliably when SZ/SZA and BP patients were considered separately. As noted, our approach was conservative in selecting eligible interactions and may have underestimated sources of shared variance (e.g., among motion-sensitive areas); nonetheless, we found the level of non-overlap striking, especially for positive symptoms, where our findings indicate an entirely distinct set of interactions governing positive-symptom like experiences in bipolar disorder and schizophrenia.

\section{Between-network connectivity abnormality is a major contributor to positive and negative symptoms}

A particularly important observation in the present study is that between-network connectivity played an essential role in estimating symptoms in every case we tested. While interactions across networks have been explored in psychotic patients ${ }^{54-56}$, prior approaches 
in the literature, including our own ${ }^{32}$, have placed more emphasis on within-network variance as the significant source of illness-related signal. In the present study, no robust relation was found between the within-network connectivity and clinical symptoms (except for the FPN). If within-network variance only reflects domain general psychopathology, and identification of domain specific biomarkers requires the assessment of variation in connectivity between networks, then our findings here indicate that accounting for individual differences in functional network boundaries is critical, as mislocalization of networks will significantly obscure the true values of low amplitude between-network correlations and hamper the discovery of brain-behavior associations (see Supplementary Fig. S9).

Additionally, psychotic symptoms appeared to be mainly related to the strengthening of between-network connectivity, although we also observed correlations with the weakening of a few connections (see Supplementary Fig. S8). To further explore this, we averaged the connectivity values of all predictive between-network connections and found that mean between-network connectivity was positively correlated with all symptoms studied here (see Supplementary Materials). The strengthening of between-network connectivity, or the loss of segregation between networks, may result from an abnormality of brain development as suggested in previous studies ${ }^{57,58}$.

\section{Structural anatomy failed to predict symptom dimensions}

Anatomical correlates of neural system integrity are generally more stable than fMRI measures, leading many in the field to focus on cortical thickness, subcortical volumes, and other neuroimaging surrogates for structural network integrity. We were unable to train any of our symptom estimation models using optimal weightings from brain structural anatomy, as derived from standardized processing pipelines (i.e. FreeSurfer). While we, like others, have identified numerous structural abnormalities in group comparisons of patient groups and healthy participants, this finding seems to indicate that structural neuroanatomy is either (a) not changing significantly as a function of specific symptom variation, or (b) our current spatial resolution of $\mathrm{T} 1$ images are insufficient to reduce measurement error into a range where they might be sensitive to symptom-specific illness-related effects.

\section{Limitations and Future Directions}

There are a few limitations that deserve mention. First, our approach identifies all connections carrying information without regard to which neural systems they comprise or any known function of such a connection, lending less obvious interpretability to our findings. That said, the distribution of informative connections revealed in our analysis did not appear to be random and was consistent with broad patterns of network-level involvement that could have tractable biological etiology. Moreover, the purpose of our study was not to make any strong claims about the nature of the underlying biology of psychotic illnesses, but rather to determine whether connectivity could provide useful information for estimating clinical state variables. Second, symptoms are still being evaluated using conventional scales, which require subjective judgment from trained clinicians. Work to provide more reliable assessments of signs and symptoms using objective behavioral recordings and real-world naturalistic measures is ongoing. Third, to date, a reliable technique for mapping functional networks in individual participants' 
subcortical regions is not yet available, precluding our ability to investigate subcortical connectivity in this study. However, we plan to adapt our individual-level approach to examine cortico-striatal connectivity in future studies, given the pivotal role of these circuits in psychiatric disorders. Finally, our analysis did not include healthy control subjects because symptom scores have little or no variation within healthy subjects, thus including them may strongly bias the symptom estimation models.

\section{Supplementary Material}

Refer to Web version on PubMed Central for supplementary material.

\section{ACKNOWLEDGMENTS}

This work was supported by NIH grants K01MH111802, K23MH104515, 1R01NS091604, P50MH106435, Beijing Municipal Science \& Technology Commission No. Z161100002616009, National Natural Science Foundation of China grants No. 81790652.

\section{REFERENCES}

1. Friston KJ, Frith CD. Schizophrenia: a disconnection syndrome? Clin Neurosci 1995; 3(2): 89-97. [PubMed: 7583624]

2. McGuire PK, Frith CD. Disordered functional connectivity in schizophrenia. Psychol Med 1996; 26(4): 663-667. [PubMed: 8817700]

3. Stephan KE, Friston KJ, Frith CD. Dysconnection in schizophrenia: from abnormal synaptic plasticity to failures of self-monitoring. Schizophr Bull 2009; 35(3): 509-527. [PubMed: 19155345]

4. Pettersson-Yeo W, Allen P, Benetti S, McGuire P, Mechelli A. Dysconnectivity in schizophrenia: Where are we now? Neurosci Biobehav Rev 2011; 35(5): 1110-1124. [PubMed: 21115039]

5. Whitfield-Gabrieli S, Ford JM. Default mode network activity and connectivity in psychopathology. Annu Rev Clin Psychol 2012; 8: 49-76. [PubMed: 22224834]

6. Whitfield-Gabrieli S, Thermenos HW, Milanovic S, Tsuang MT, Faraone SV, McCarley RW et al. Hyperactivity and hyperconnectivity of the default network in schizophrenia and in first-degree relatives of persons with schizophrenia. Proc Natl Acad Sci U S A 2009; 106(4): 1279-1284. [PubMed: 19164577]

7. Anticevic A, Cole MW, Repovs G, Murray JD, Brumbaugh MS, Winkler AM et al. Characterizing Thalamo-Cortical Disturbances in Schizophrenia and Bipolar Illness. Cereb Cortex 2013.

8. Woodward ND, Rogers B, Heckers S. Functional resting-state networks are differentially affected in schizophrenia. Schizophr Res 2011; 130(1-3): 86-93. [PubMed: 21458238]

9. Chen YL, Tu PC, Lee YC, Chen YS, Li CT, Su TP. Resting-state fMRI mapping of cerebellar functional dysconnections involving multiple large-scale networks in patients with schizophrenia. Schizophr Res 2013; 149(1-3): 26-34. [PubMed: 23810119]

10. Cole MW, Anticevic A, Repovs G, Barch D. Variable global dysconnectivity and individual differences in schizophrenia. Biol Psychiatry 2011; 70(1): 43-50. [PubMed: 21496789]

11. Shenton ME, Dickey CC, Frumin M, McCarley RW. A review of MRI findings in schizophrenia. Schizophr Res 2001; 49(1): 1-52.

12. Hoptman MJ, Antonius D, Mauro CJ, Parker EM, Javitt DC. Cortical thinning, functional connectivity, and mood-related impulsivity in schizophrenia: relationship to aggressive attitudes and behavior. Am J Psychiatry 2014; 171(9): 939-948. [PubMed: 25073506]

13. Walton E, Hibar DP, van Erp TGM, Potkin SG, Roiz-Santianez R, Crespo-Facorro B et al. Prefrontal cortical thinning links to negative symptoms in schizophrenia via the ENIGMA consortium. Psychol Med 2017: 1-13. 
14. Walton E, Hibar DP, van Erp TG, Potkin SG, Roiz-Santianez R, Crespo-Facorro B et al. Positive symptoms associate with cortical thinning in the superior temporal gyrus via the ENIGMA Schizophrenia consortium. Acta Psychiatr Scand 2017; 135(5): 439-447. [PubMed: 28369804]

15. Lui S, Deng W, Huang X, Jiang L, Ma X, Chen H et al. Association of cerebral deficits with clinical symptoms in antipsychotic-naive first-episode schizophrenia: an optimized voxel-based morphometry and resting state functional connectivity study. Am J Psychiatry 2009; 166(2): 196205. [PubMed: 18981063]

16. Anticevic A, Brumbaugh MS, Winkler AM, Lombardo LE, Barrett J, Corlett PR et al. Global prefrontal and fronto-amygdala dysconnectivity in bipolar I disorder with psychosis history. Biol Psychiatry 2013; 73(6): 565-573. [PubMed: 22980587]

17. Thomas Yeo BT, Krienen FM, Sepulcre J, Sabuncu MR, Lashkari D, Hollinshead M et al. The organization of the human cerebral cortex estimated by intrinsic functional connectivity. $\mathrm{J}$ Neurophysiol 2011; 106(3): 1125-1165. [PubMed: 21653723]

18. Laumann TO, Gordon EM, Adeyemo B, Snyder AZ, Joo SJ, Chen MY et al. Functional System and Areal Organization of a Highly Sampled Individual Human Brain. Neuron 2015; 87(3): 657670. [PubMed: 26212711]

19. Gordon EM, Laumann TO, Gilmore AW, Newbold DJ, Greene DJ, Berg JJ et al. Precision Functional Mapping of Individual Human Brains. Neuron 2017; 95(4): 791-807.e797. [PubMed: 28757305]

20. Glasser MF, Coalson TS, Robinson EC, Hacker CD, Harwell J, Yacoub E et al. A multi-modal parcellation of human cerebral cortex. Nature 2016; 536(7615): 171-178. [PubMed: 27437579]

21. Power JD, Cohen AL, Nelson SM, Wig GS, Barnes KA, Church JA et al. Functional network organization of the human brain. Neuron 2011; 72(4): 665-678. [PubMed: 22099467]

22. Wig GS, Laumann TO, Cohen AL, Power JD, Nelson SM, Glasser MF et al. Parcellating an individual subject's cortical and subcortical brain structures using snowball sampling of restingstate correlations. Cereb Cortex 2014; 24(8): 2036-2054. [PubMed: 23476025]

23. Choi EY, Yeo BT, Buckner RL. The organization of the human striatum estimated by intrinsic functional connectivity. J Neurophysiol 2012; 108(8): 2242-2263. [PubMed: 22832566]

24. Spronk M, Ji JL, Kulkarni K, Repovs G, Anticevic A, Cole MW. Mapping the human brain's cortical-subcortical functional network organization. bioRxiv 2018: 206292.

25. Kraguljac NV, White DM, Hadley N, Hadley JA, ver Hoef L, Davis E et al. Aberrant hippocampal connectivity in unmedicated patients with schizophrenia and effects of antipsychotic medication: a longitudinal resting state functional MRI study. Schizophr Bull 2016; 42(4): 1046-1055. [PubMed: 26873890]

26. Lui S, Deng W, Huang X, Jiang L, Ma X, Chen H et al. Association of cerebral deficits with clinical symptoms in antipsychotic-naive first-episode schizophrenia: an optimized voxel-based morphometry and resting state functional connectivity study. Am J Psychiatry 2009; 166(2): 196205. [PubMed: 18981063]

27. Mueller S, Wang D, Fox MD, Yeo BT, Sepulcre J, Sabuncu MR et al. Individual variability in functional connectivity architecture of the human brain. Neuron 2013; 77(3): 586-595. [PubMed: 23395382]

28. Langs G, Wang D, Golland P, Mueller S, Pan R, Sabuncu MR et al. Identifying Shared Brain Networks in Individuals by Decoupling Functional and Anatomical Variability. Cereb Cortex 2015.

29. Braga RM, Buckner RL. Parallel Interdigitated Distributed Networks within the Individual Estimated by Intrinsic Functional Connectivity. Neuron 2017; 95(2): 457-471.e455. [PubMed: 28728026]

30. Wang D, Buckner RL, Fox MD, Holt DJ, Holmes AJ, Stoecklein S et al. Parcellating cortical functional networks in individuals. Nat Neurosci 2015; 18(12): 1853-1860. [PubMed: 26551545]

31. Van Dijk KR, Sabuncu MR, Buckner RL. The influence of head motion on intrinsic functional connectivity MRI. Neuroimage 2012; 59(1): 431-438. [PubMed: 21810475]

32. Baker JT, Holmes AJ, Masters GA, Yeo BT, Krienen F, Buckner RL et al. Disruption of cortical association networks in schizophrenia and psychotic bipolar disorder. JAMA Psychiatry 2014; 71(2): 109-118. [PubMed: 24306091] 
33. Buckner RL, Krienen FM, Castellanos A, Diaz JC, Yeo BT. The organization of the human cerebellum estimated by intrinsic functional connectivity. J Neurophysiol 2011; 106(5): 23222345. [PubMed: 21795627]

34. Yeo BT, Krienen FM, Sepulcre J, Sabuncu MR, Lashkari D, Hollinshead M et al. The organization of the human cerebral cortex estimated by intrinsic functional connectivity. J Neurophysiol 2011; 106(3): 1125-1165. [PubMed: 21653723]

35. Wang D, Buckner RL, Fox MD, Holt DJ, Holmes AJ, Stoecklein S et al. Parcellating cortical functional networks in individuals. Nat Neurosci 2015; 18(12): 1853. [PubMed: 26551545]

36. Glasser MF, Coalson TS, Robinson EC, Hacker CD, Harwell J, Yacoub E et al. A multi-modal parcellation of human cerebral cortex. Nature 2016; 536(7615): 171-178. [PubMed: 27437579]

37. Lui S, Zhou XJ, Sweeney JA, Gong Q. Psychoradiology: the frontier of neuroimaging in psychiatry. Radiology 2016; 281(2): 357-372. [PubMed: 27755933]

38. Sun H, Chen Y, Huang Q, Lui S, Huang X, Shi Y et al. Psychoradiologic utility of MR imaging for diagnosis of attention deficit hyperactivity disorder: a radiomics analysis. Radiology 2017; 287(2): 620-630. [PubMed: 29165048]

39. Port JD. Diagnosis of Attention Deficit Hyperactivity Disorder by Using MR Imaging and Radiomics: A Potential Tool for Clinicians. Radiology 2018; 287: 631-632. [PubMed: 29668406]

40. Kuhn S, Musso F, Mobascher A, Warbrick T, Winterer G, Gallinat J. Hippocampal subfields predict positive symptoms in schizophrenia: first evidence from brain morphometry. Transl Psychiatry 2012; 2: e127. [PubMed: 22692142]

41. Rotarska-Jagiela A, Oertel-Knoechel V, DeMartino F, van de Ven V, Formisano E, Roebroeck A et al. Anatomical brain connectivity and positive symptoms of schizophrenia: a diffusion tensor imaging study. Psychiatry Res 2009; 174(1): 9-16. [PubMed: 19767179]

42. Seok JH, Park HJ, Chun JW, Lee SK, Cho HS, Kwon JS et al. White matter abnormalities associated with auditory hallucinations in schizophrenia: a combined study of voxel-based analyses of diffusion tensor imaging and structural magnetic resonance imaging. Psychiatry Res 2007; 156(2): 93-104. [PubMed: 17884391]

43. Cheung V, Chiu CP, Law CW, Cheung C, Hui CL, Chan KK et al. Positive symptoms and white matter microstructure in never-medicated first episode schizophrenia. Psychol Med 2011; 41(8): 1709-1719. [PubMed: 20809999]

44. Goghari VM, Sponheim SR, MacDonald AW. The Functional Neuroanatomy of Symptom Dimensions in Schizophrenia: A Qualitative and Quantitative Review of a Persistent Question. Neurosci Biobehav Rev 2010; 34(3): 468. [PubMed: 19772872]

45. Khadka S, Meda SA, Stevens MC, Glahn DC, Calhoun VD, Sweeney JA et al. Is Aberrant Functional Connectivity A Psychosis Endophenotype? A Resting State Functional Magnetic Resonance Imaging Study. Biol Psychiatry 2013; 74(6): 458-466. [PubMed: 23746539]

46. Meda SA, Gill A, Stevens MC, Lorenzoni RP, Glahn DC, Calhoun VD et al. Differences in restingstate fMRI functional network connectivity between schizophrenia and psychotic bipolar probands and their unaffected first-degree relatives. Biol Psychiatry 2012; 71(10): 881-889. [PubMed: 22401986]

47. Benoit A, Bodnar M, Malla AK, Joober R, Lepage M. The structural neural substrates of persistent negative symptoms in first-episode of non-affective psychosis: a voxel-based morphometry study. Front Psychiatry 2012; 3: 42. [PubMed: 22586412]

48. Li Y, Li WX, Xie DJ, Wang Y, Cheung EF, Chan RC. Grey matter reduction in the caudate nucleus in patients with persistent negative symptoms: An ALE meta-analysis. Schizophr Res 2017.

49. Kim G-W, Kim Y- H, Jeong G- W. Whole brain volume changes and its correlation with clinical symptom severity in patients with schizophrenia: A DARTEL-based VBM study. PLoS One 2017; 12(5): e0177251. [PubMed: 28520743]

50. Adler CM, Adams J, DelBello MP, Holland SK, Schmithorst V, Levine A et al. Evidence of white matter pathology in bipolar disorder adolescents experiencing their first episode of mania: a diffusion tensor imaging study. Am J Psychiatry 2006; 163(2): 322-324. [PubMed: 16449490]

51. Martino M, Magioncalda P, Saiote C, Conio B, Escelsior A, Rocchi G et al. Abnormal functionalstructural cingulum connectivity in mania: combined functional magnetic resonance imaging- 
diffusion tensor imaging investigation in different phases of bipolar disorder. Acta Psychiatr Scand 2016; 134(4): 339-349. [PubMed: 27273612]

52. Pomarol-Clotet E, Moro N, Sarro S, Goikolea JM, Vieta E, Amann B et al. Failure of de-activation in the medial frontal cortex in mania: evidence for default mode network dysfunction in the disorder. World J Biol Psychiatry 2012; 13(8): 616-626. [PubMed: 21604958]

53. Li M, Huang C, Deng W, Ma X, Han Y, Wang Q et al. Contrasting and convergent patterns of amygdala connectivity in mania and depression: a resting-state study. J Affect Disord 2015; 173: 53-58. [PubMed: 25462396]

54. Çetin MS, Christensen F, Abbott CC, Stephen JM, Mayer AR, Cañive JM et al. Thalamus and posterior temporal lobe show greater inter-network connectivity at rest and across sensory paradigms in schizophrenia. Neuroimage 2014; 97: 117-126. [PubMed: 24736181]

55. Bassett DS, Nelson BG, Mueller BA, Camchong J, Lim KO. Altered resting state complexity in schizophrenia. Neuroimage 2012; 59(3): 2196-2207. [PubMed: 22008374]

56. Repovs G, Csernansky JG, Barch DM. Brain network connectivity in individuals with schizophrenia and their siblings. Biol Psychiatry 2011; 69(10): 967-973. [PubMed: 21193174]

57. Satterthwaite TD, Vandekar SN, Wolf DH, Bassett DS, Ruparel K, Shehzad Z et al. Connectomewide network analysis of youth with Psychosis-Spectrum symptoms. Mol Psychiatry 2015; 20(12): 1508. [PubMed: 26033240]

58. Fair DA, Cohen AL, Power JD, Dosenbach NU, Church JA, Miezin FM et al. Functional brain networks develop from a "local to distributed" organization. PLoS Comput Biol 2009; 5(5): e1000381. [PubMed: 19412534] 
a

C b
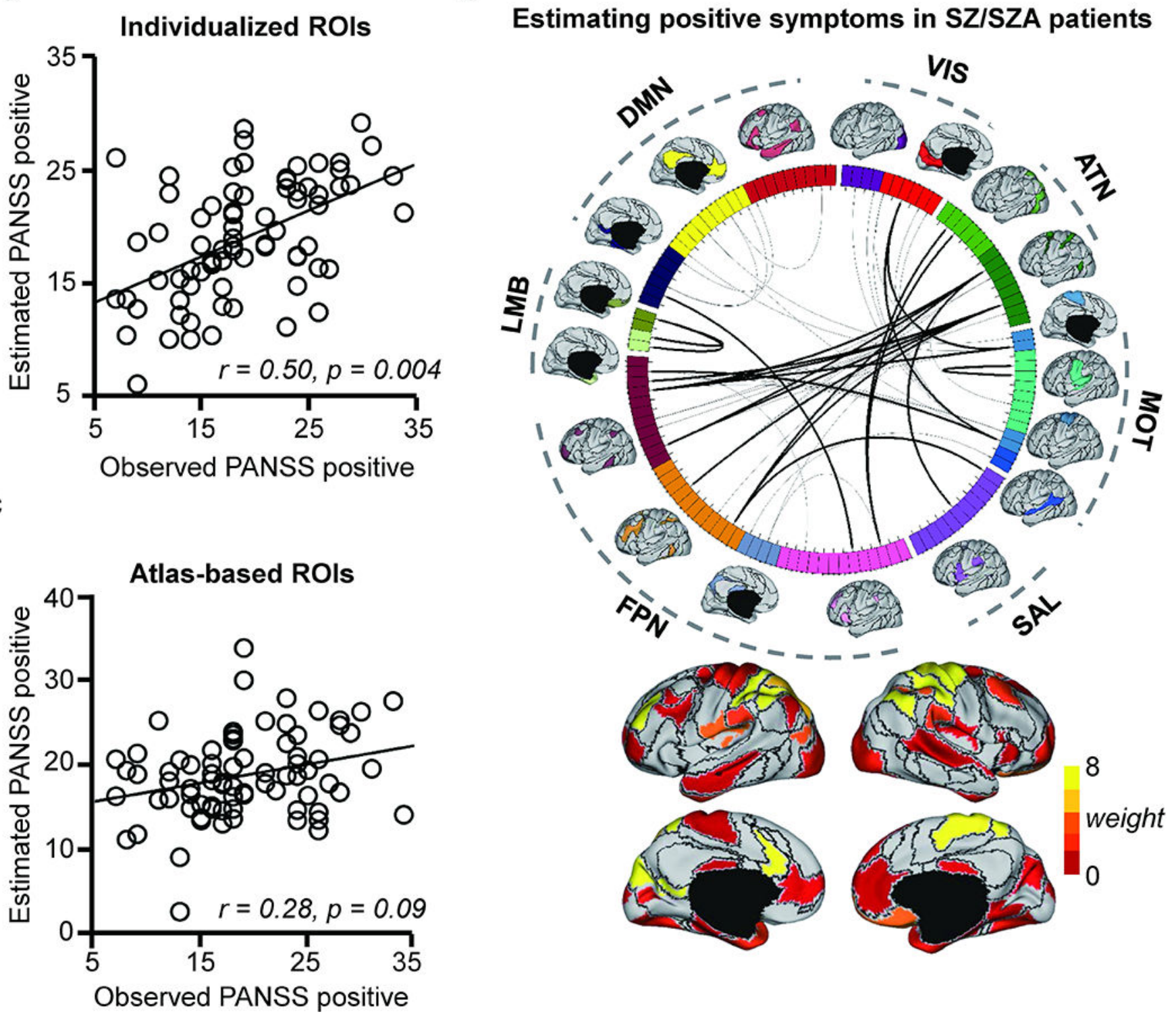

Fig. 1.

Positive symptoms in patients with schizophrenia spectrum illnesses can be predicted by functional connectivity after accounting for individual variation in functional region distribution and head motion. (a) The scatterplot illustrates the correlation $(r=0.50, p=$ 0.004 , permutation test) between the PANSS positive scores predicted by connectivity among the individually-specified ROIs and the scores that were actually observed in the 76 patients with SZ or SZA. (b) 116 ROIs extracted from 18 individualized networks are represented on a wheel. Fifty-two connections that are predictive of the PANSS positive scores are plotted (top 20 connections are indicated by the dark lines). Group-level maps of the 18 functional networks are shown outside the wheel. The 116 ROIs are color-coded according to 7 canonical networks: visual (VIS), sensorimotor (MOT), attention (ATN), salience (SAL), limbic (LMB), frontopariental control (FPN) and the default network (DN). Functional regions involved in the most predictive connections are rendered on the cortical 
surface. The predictive power of each connection is indicated by the "weight", as colorcoded on the cortical surface. (c) A similar analysis is performed using 116 ROIs defined in a group-level network template. Functional connectivity based on the brain template is not able to predict the PANSS positive scores in these patients $(r=0.28, p=0.09$, permutation test). 
a

C b
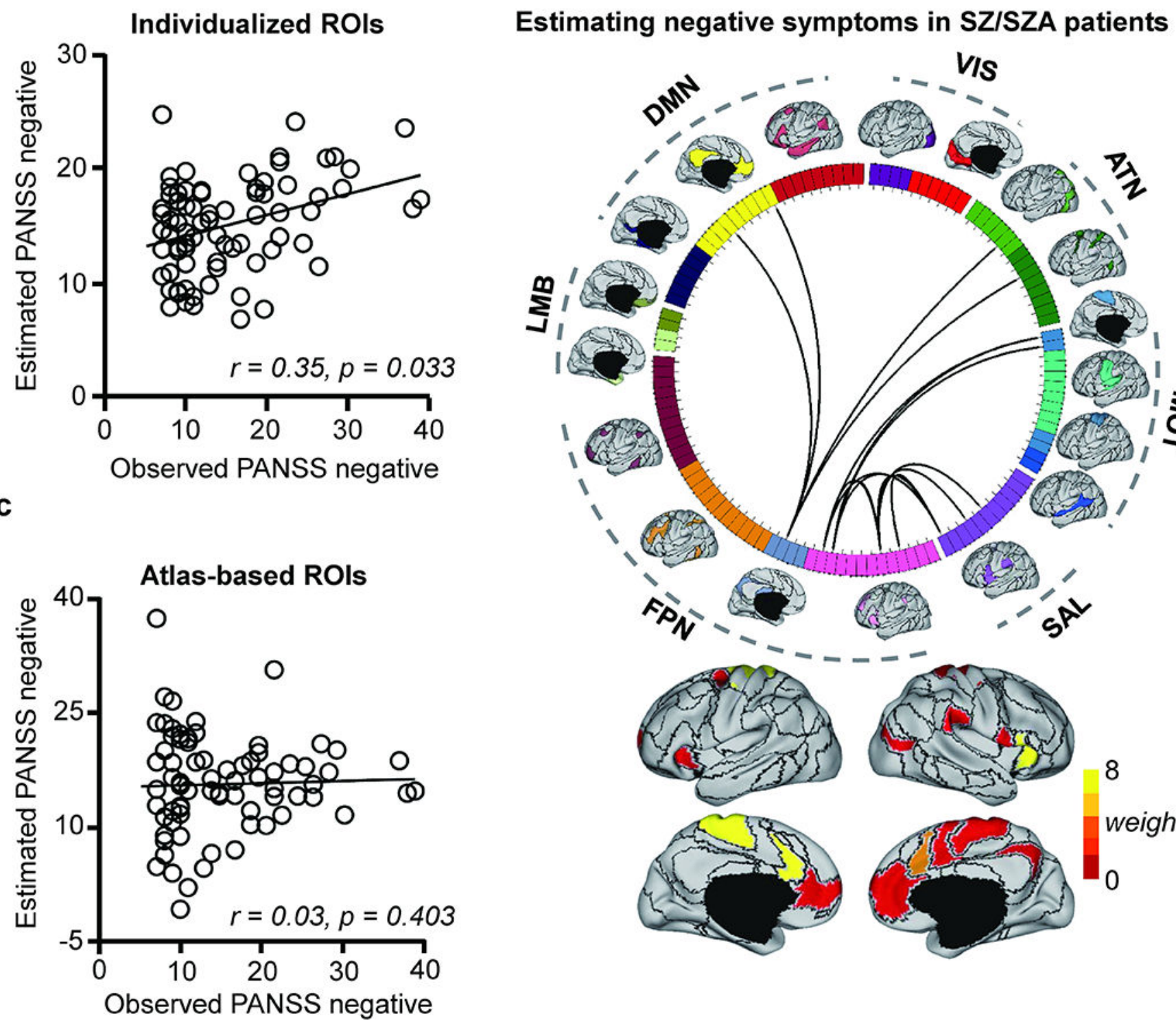

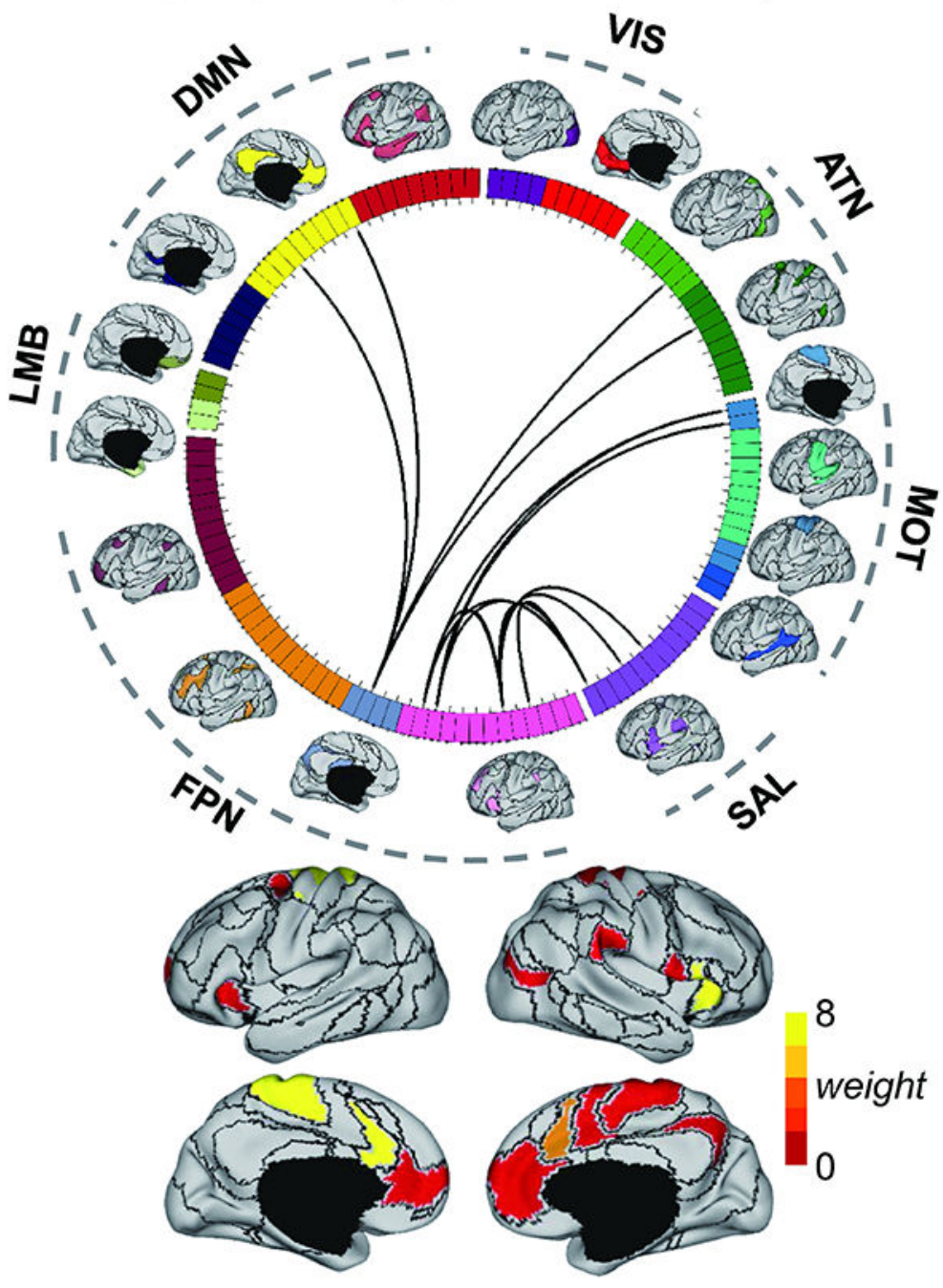

Fig. 2.

(a) Functional connectivity among the subject-specific ROIs can predict the PANSS negative subscale scores in 76 patients with schizophrenia or schizoaffective disorder. The scatterplot illustrates the correlation ( $r=0.35, p=0.033$, permutation test) between the predicted and observed PANSS negative scores. (b) Fifteen connections that are predictive of the PANSS negative scores are plotted on the wheel. Functional regions involved in the most predictive connections are rendered on the cortical surface. (c) A similar analysis is performed using 116 ROIs defined in a group-level network template. Functional connectivity based on the brain template is not able to predict the PANSS negative scores in these patients ( $r=0.03, p$ $=0.403$, permutation test . 

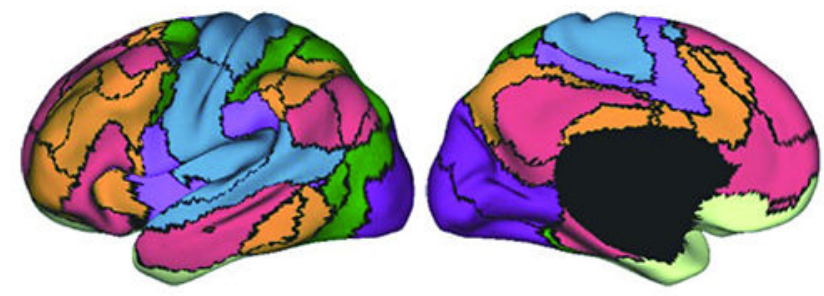

$\square$ Between-network Within-network

a

\section{Predicting positive symptoms}

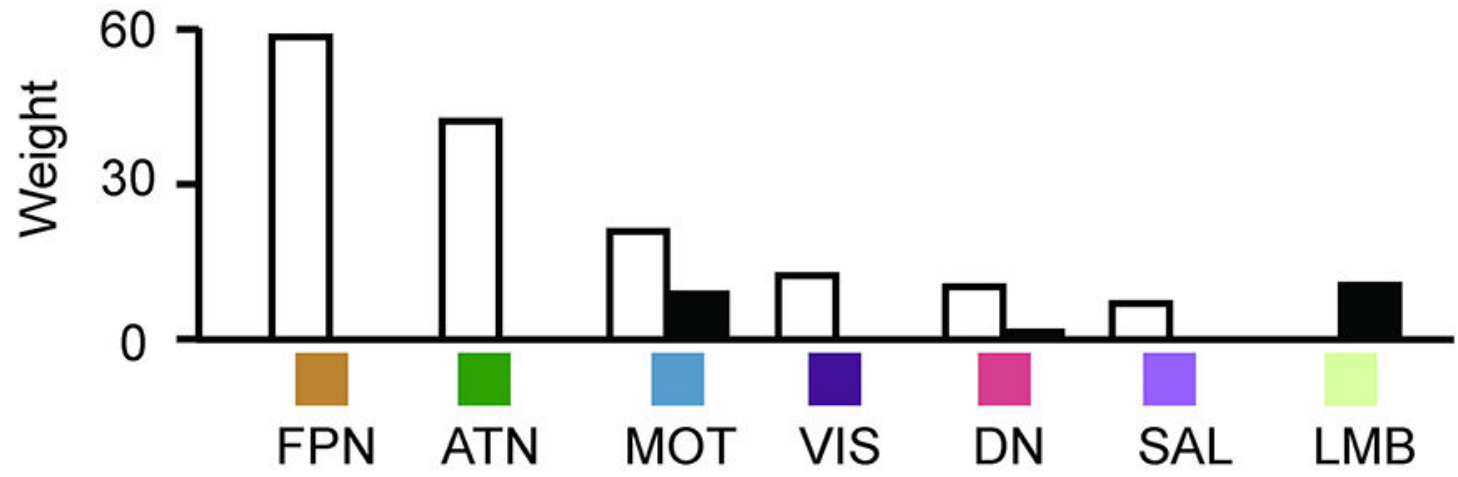

b

\section{Predicting negative symptoms}

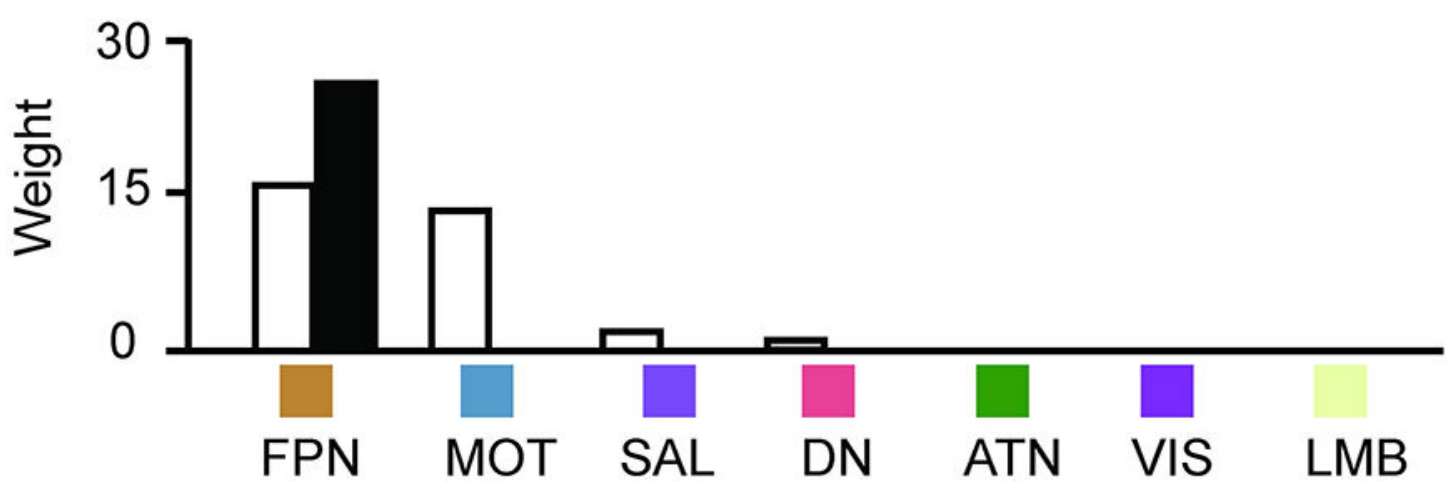

Fig. 3.

Between-network connectivity plays an essential role in predicting the positive and negative symptoms in patients with schizophrenia spectrum illness. (a) The functional connections most predictive of the symptom severity scores in SZ/SZA patients are grouped according to the 7 canonical networks. Connections contributing to the positive symptom prediction are mainly between-network connections (white bars). These between-network connections mainly involve the FPN, ATN and MOT. (b). Connections contributing to the negative symptom prediction mainly involve between-network connectivity in the FPN and MOT. Connections within the FPN also contributed to the prediction. 
a

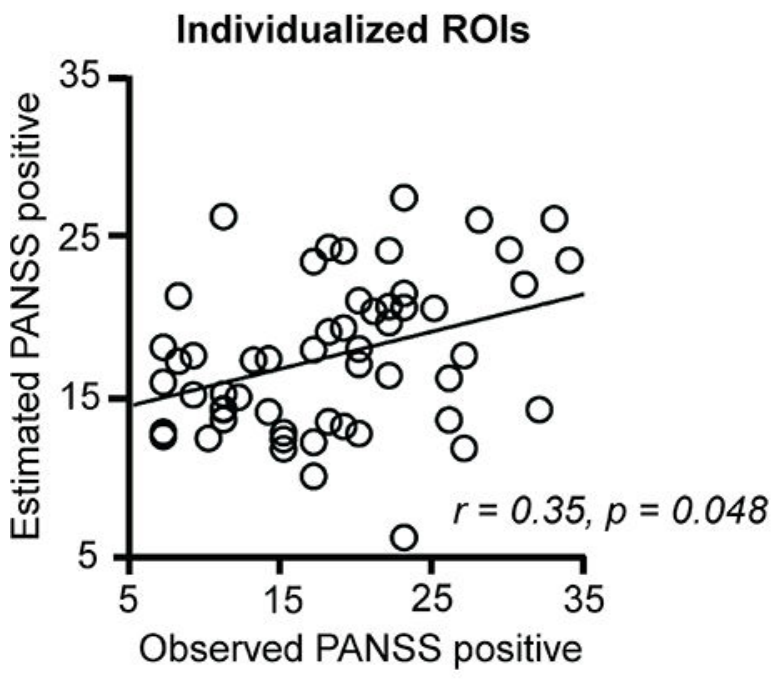

C

Atlas-based ROls

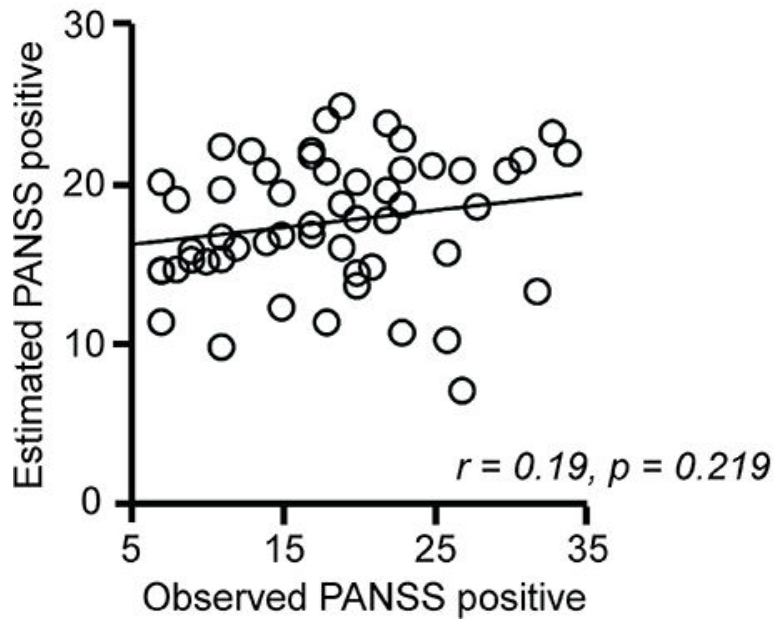

b
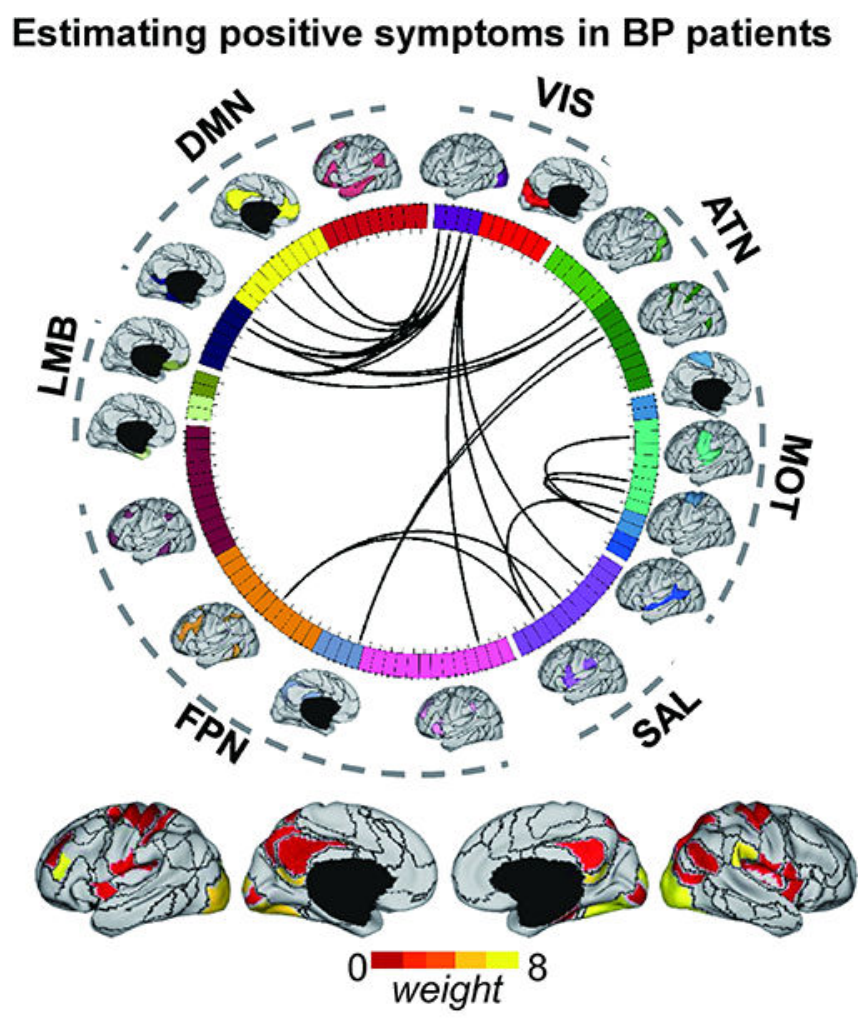

Weight in prediction

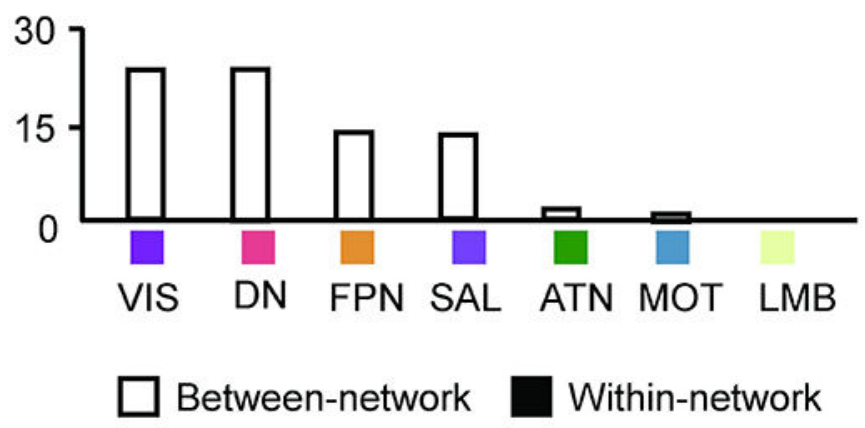

Fig. 4.

(a) Functional connectivity among the subject-specific ROIs can predict the PANSS positive scores in 55 patients with bipolar disorder. The predicted and observed PANSS positive scores are significantly correlated ( $r=0.35, p=0.048$, permutation test). (b) Twenty-six connections that are predictive of the PANSS positive scores are plotted on the wheel. (c) Functional connectivity based on the brain template is not able to predict the PANSS positive scores in these patients ( $r=0.19, p=0.219$, permutation test). (d) The prediction 
based on subject-specific ROIs is also driven by between-network connectivity involving the SAL, DN, VIS, and FPN. 


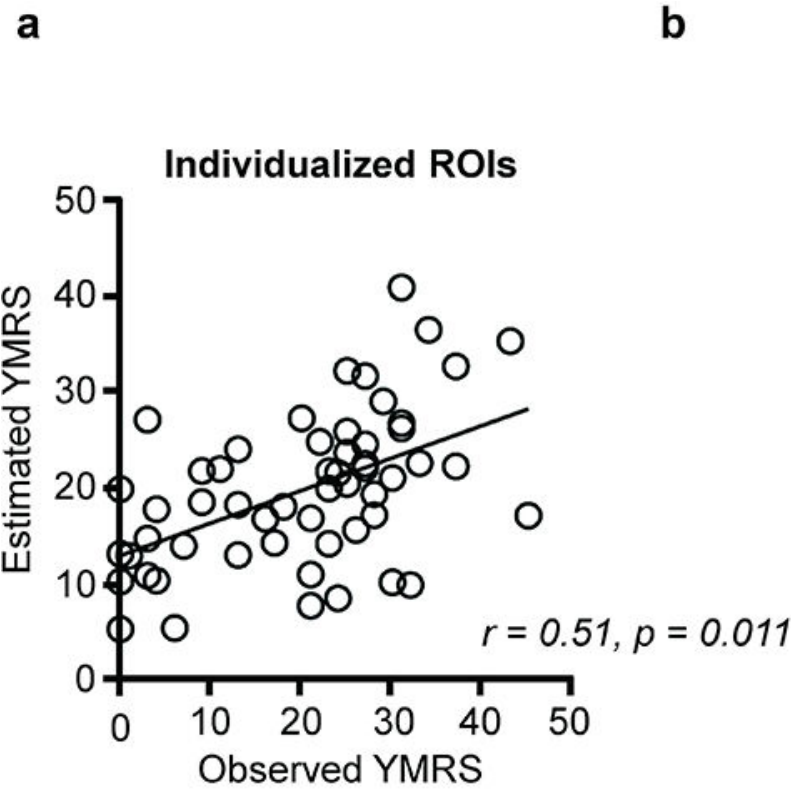

C

Atlas-based ROls

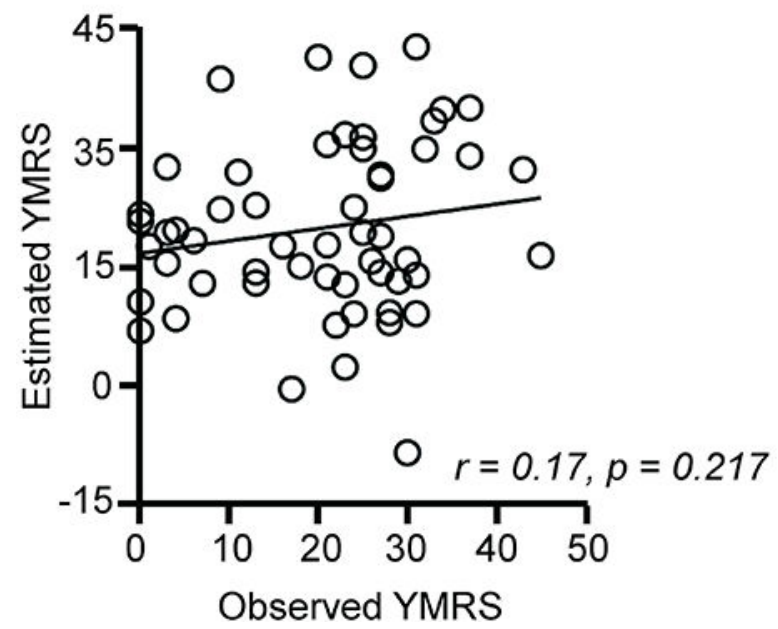

d

\section{Estimating mania score in BP patients}

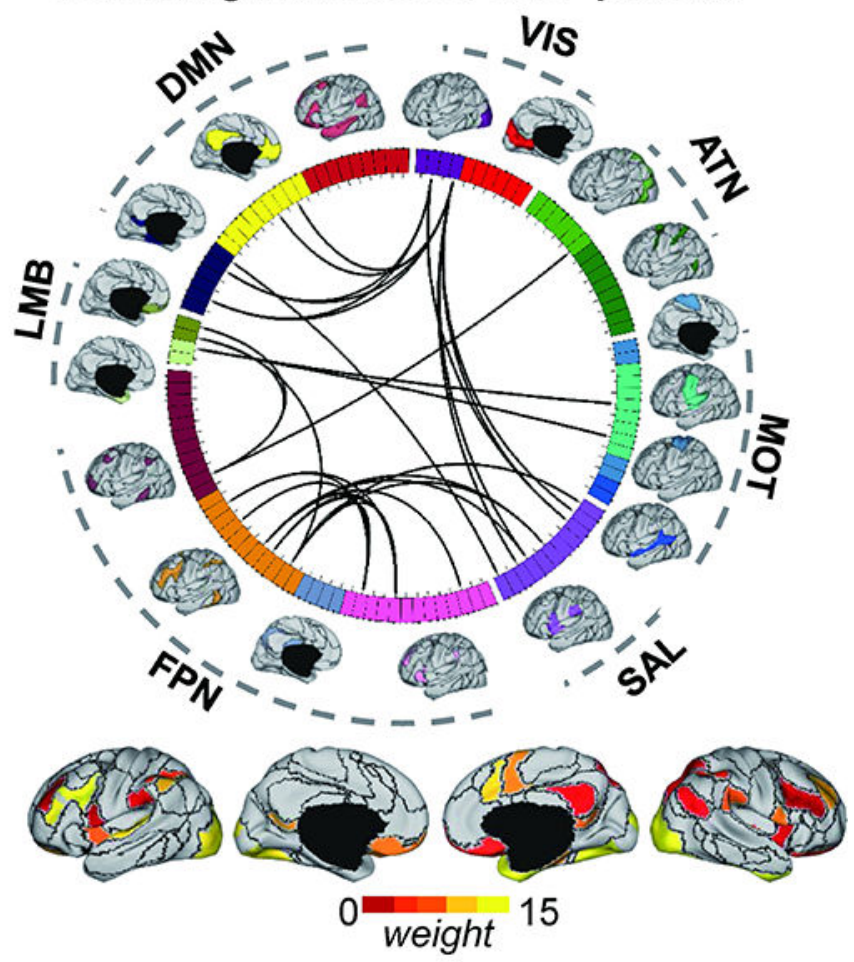

Weight in prediction

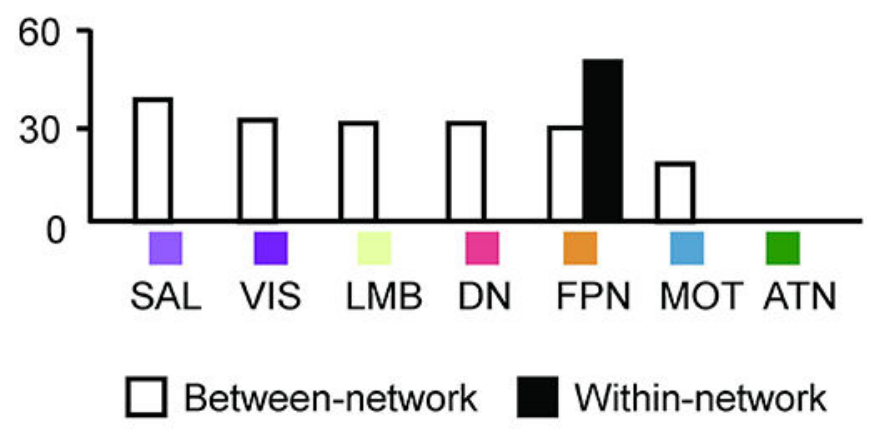

Fig. 5.

(a) Functional connectivity among the subject-specific ROIs can predict the mania score (YMRS) in patients with bipolar disorder $(r=0.51, p=0.011$, permutation test). (b) Thirtytwo connections that are predictive of the PANSS positive scores are plotted on the wheel.

(c) Functional connectivity based on the brain template is less predictive of the mania score ( $r=0.17, p=0.217$, permutation test). (d) The prediction is mainly driven by between- 
network connections involving the SAL, VIS, LMB and DN. Connections within the FPN also contributed to the prediction. 